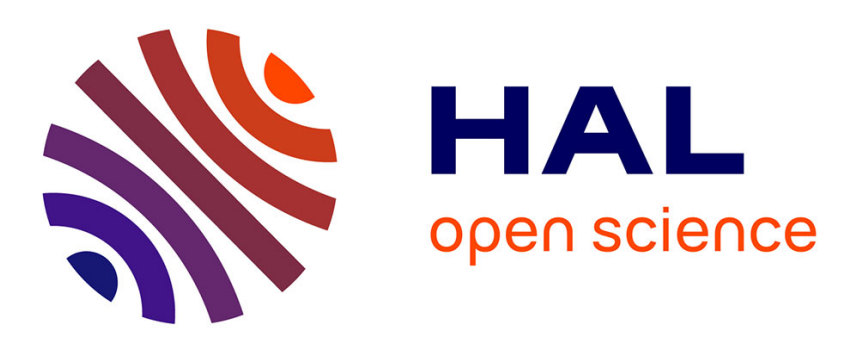

\title{
Segmentation of anatomical structures from 3D brain MRI using automatically-built statistical shape models
}

\author{
Jonathan Bailleul, Su Ruan, Daniel Bloyet, Barbara Romaniuk
}

\section{To cite this version:}

Jonathan Bailleul, Su Ruan, Daniel Bloyet, Barbara Romaniuk. Segmentation of anatomical structures from 3D brain MRI using automatically-built statistical shape models. IEEE International Conference on Image Processing (ICIP'04), 2004, Singapore, Singapore. pp.2741-2744, 10.1109/ICIP.2004.1421671 . hal-00844015

\section{HAL Id: hal-00844015 https://hal.science/hal-00844015}

Submitted on 2 Apr 2015

HAL is a multi-disciplinary open access archive for the deposit and dissemination of scientific research documents, whether they are published or not. The documents may come from teaching and research institutions in France or abroad, or from public or private research centers.
L'archive ouverte pluridisciplinaire HAL, est destinée au dépôt et à la diffusion de documents scientifiques de niveau recherche, publiés ou non, émanant des établissements d'enseignement et de recherche français ou étrangers, des laboratoires publics ou privés. 


\title{
SEGMENTATION OF ANATOMICAL STRUCTURES FROM 3D BRAIN MRI USING AUTOMATICALLY-BUILT STATISTICAL SHAPE MODELS
}

\author{
Jonathan Bailleul, Su Ruan, Daniel Bloyet, Barbara Romaniuk \\ GREYC CNRS UMR 6072, Ensicaen \& University of Caen, F-14050 Caen cedex, France \\ \{Jonathan.Bailleul — Su.Ruan — Daniel.Bloyet\}@ greyc.ismra.fr
}

\begin{abstract}
We propose a twofold method that first automatically builds a statistical shape model of anatomical 3D brain structures of interest, then uses this model for delineating structure contours onto any patient MRI. First of all, an estimated training set of shapes is infered by registration of a 3D anatomical atlas over a set of brain MRIs, then automatically landmarked using the "Minimum Description Length" based method developed by Davies et al.[4]. A 3D "Point Distribution Model" is then established and used to constrain the delineation process. It is lead by a novel intensity model specifically developed to overcome the estimated nature of our training set in exploiting only local intensities.
\end{abstract}

\section{INTRODUCTION}

Neuro-imaging praticians currently need tools to perform delineation of structures of interest (i.e the accurate determination of contour) over large 3D brain MRIs sets in order to help several studies going from functional brain mapping to data-mining applications. Correlations are sought between structure and patient characteristics like pathology, handedness or gender.

Automation is necessary to cope with the amount of data to process and with the low quality of images: human experts require a lot of time to segment only one structure onto one MRI, and will inherently introduce variability in their decisions. Furthermore, structures of interest show weak contrast and high noise at boundaries since they are made of anatomical tissues mixtures: Cerebro-Spinal Fluid, Grey Matter and White Matter.

Our first approach revamped a region-based method[9] developed in our team by J.H.Xue, where small homogeneous regions were progressively accumulated to build structures, accounting for a priori information derived into fuzzy sets from an anatomical atlas Atlas ${ }_{a}$ (cf 2.2).

Nevertheless, relevant accuracy at contours was still randomly achieved since this bottom-up approach, willing to leave room for shape variability, modeled quite loosely the geometric information from Atlas . Though, Bloch et al.[2] achieved excellent results with another fuzzy approach based upon quite different assumptions. But for now, it still needs to follow a precise path for segmenting structures and cannot strictly ensure shape consistency of the result.

To overcome this limitation, we selected the statistical Point Distribution Model (PDM) from Cootes, Taylor et al. [3] that considers a mean shape and its allowed linear deformation modes, both inferred from a training set of valid shapes annotated by corresponding landmark points (cf. 2.1). Their delineation method, named Active Shape Model (ASM, or smart snake), is lead by a statistical Intensity Model of the gray level environment around each landmark over training set MRIs, and constrained by the PDM Shape Model to only raise consistent results. This top-down approach widely proved its efficiency in medical imaging (e.g [7][5]), but becomes really demanding in $3 \mathrm{D}$ as it requires delineated and landmarked shapes.

For this reason, every considered 3D ASM implementation started from a set of structure contours provided by fellow experts, hence each often concerns few structures. The second challenge, namely automatic landmarking, is frequently handled with arbitrament due to the difficulty to formalize an inherently intuitive process.

Former methods like [6] are restricted to compound tubular shapes so as to decompose the problem in several affordable 2D PDMs. Frangi et al. [5] designate noticeable points in decimating a mean volumic shape, then establish correspondence through warp-registering these over the training set. Kelemen et al. [7] obtain correspondence in projecting parameterized shapes surfaces into an object-centered coordinate system, where uniform sampling finally raises some landmarks. Though these methods produce functional PDMs through reasonable assumptions, none might warranty their optimality.

Davies et al.[4] recently managed to establish an automated and objective landmarking method using simplex optimization framework guided by an objective function estimating PDM relevance in exploiting the Minimum Descrip- 
tion Length (MDL) principle. This exciting contribution finally enables us to propose a novel ASM method.

\subsection{Proposed Method}

We first propose a method that can automatically build a single PDM Shape Model for any structure contained in Atlas $_{a}$ over any MRI population. Training set delineation is performed through Atlas $_{a}$ registration over selected MRIs, an arguable choice that we expressly defend. Landmarking is then performed by the optimal MDL method[4].

We also propose a new Intensity Model that will fit the constrains of our training set to guide delineation without statistical priors in accurately exploiting subtle local changes in the current $M r i_{\lambda}$ around the boundary.

\section{AUTOMATIC 3D PDM BUILDING IN BRAIN MRI}

\subsection{Point Distribution Model Principle}

Each shape of the training set must be annotated by $n_{l}$ landmarks, each designating the same anatomical locus along the set. The set is considered as a collection of shape vectors $\left\{s_{i}\right\}_{\left(i \in\left[1, n_{s}\right]\right)}$ (with $s_{i}=\left\{x_{1}, y_{1}, z_{1} \ldots x_{n_{l}}, y_{n_{l}}, z_{n_{l}}\right\}$ ), which after alignment raises a covariance matrix.

Principal Component Analysis (PCA) provides $n_{p} n_{p}$-D eigenvectors describing linear variation modes infered from $\left\{s_{i}\right\}$ and sorted by corresponding eigenvalues $\lambda_{m}$ :

$$
s_{i}=\bar{s}+\sum_{m=1}^{n_{p}} p^{m} b_{i}^{m}
$$

The PDM formulation (Eq. 1) defines an Allowable Shape Domain (ASD) centered on mean shape $\bar{s}$ as the set of linear combinations of significant eigenvectors ponderated by shape coefficients $b_{i}^{m}$, bounded by $+/-3 \lambda_{m}$ (details in [3]).

\subsection{Automatic Estimated Training Set Building}

Obtaining a delineated training set is quite straightforward in $2 \mathrm{D}$, since it generally requires one slice over about 20 patients (cf [3][4]). In 3D, the increased shape complexity and variability requires more instances and, for each structure, about 15 slices per volume.

Thus, when a team of experts achieve such training sets through many efforts, these cover just a few specific structures over a given MRI population. Mixing such results to increase the structure number is difficult since populations might be inconsistent (eg, significant differences appear with age on ventricles). But the main problem is that experts tend to restrict use and diffusion of their sets to their own research teams.
Using experience acquired in our previous work[9], we considered Atlas $s_{a}$, i.e. the result of an expert delineation of structures on a reference MRI, and decided to warp-register (AIR) it over a mixed set of 30 patient MRIs selected among the wide pool available from Cyceron center. Though arbitrary and rather simplistic, this method eventually appears both automatic and consistent:

- We can finally work with any structure featured in any atlas. Harvard SPL \& Cyceron already contain all structures of interest, and adding one to selected atlas, if necessary, will require affordable work ( $\simeq 20$ slices).

- Difference between expected and obtained contours appears really acceptable (within 2 voxels) after many visual checks. This is helped by the global nature of the registration and also the favorable configuration of our structures[1].

- Delineated instances have the same shape as Atlas $s_{a}$ reference, since they derive from it through (global) warp deformation.

- Though, they show obvious variations over the selected MRIs

It appears that the conditions required to build a relevant Shape Model - namely, numerous correct shapes and likely variability - are fulfilled. Nevertheless, the real cost of this assumption is that exact matching between delineation and MRIs is broken, which will disrupt learning of a precise gray-level environment (more details in [1]).

\subsection{Automatic Training Set Landmarking}

Landmarking a training set of delineated shape instances of a single structure consists in inferring both the mean shape geometry and the ways it varies along the set: landmarks are shape invariants. This quite intuitive process can be affordable in $2 \mathrm{D}$, but no longer in $3 \mathrm{D}$ where shape complexity and variability invalidate any intuition. Automation of this usually manual process represented for long the major challenge for extending PDMs to 3D.

Davies et al.[4] recently developed a method that performs automatic landmarking through a Neder-Mead Simplex optimization process ensuring good confidence in the optimality of resulting PDM. Consequences are twofold:

- Landmarking hypothesis must be parameterized to enable the simplex to steer the optimization. This is achieved in expressing reparameterizations of landmarked unit spheres using Cauchy Kernels, the landmarks being back-projected to the set via amended conformal mapping between each instance and the unit sphere.

- The corresponding PDM relevance must be evaluated to enable the simplex to reach the best annotation hypothesis: this is the major contribution of Davies et al. Following the MDL principle, the PDM model is considered 
as a discrete message encoding both parameters and data. Minimizing the PDM's MDL, the simplex will attempt to improve both PDM compactness and accuracy, preserving its generalization ability balanced by correctness. Full details are reviewed in [4].

In our method[1], we used the method of Davies et al.[4] implemented by Kildeby et al.[8].

\section{A NEW ASM INTENSITY MODEL}

The Active Shape Model (ASM) delineation procedure, established in [3], primarily consists in a dialog between an Intensity Model and a Shape Model. First, a mean shape $\bar{s}$ is approximately posed into the target image. Then, the Intensity Model proposes moves for each of its landmark along their normal direction so as to better fit the image. Nevertheless, noisy and low-contrasted borders often occur in medical imaging, causing incorrect proposals. The purpose of the Shape Model is to examine and amend these move proposals if they threaten shape integrity, namely if the shape escapes from the ASD.

To ensure final success, the move amplitude is restricted and dialogs between both models are iterated until eventual idempotence, that is assumed to raise an optimal delineation result. Nevertheless, even if a correct PDM is required to regulate the process, it is primarily driven by the selected Intensity Model.

\subsection{Standard Framework}

Though 2D/3D ASM implementations might vary significantly in the way both models communicate or in the amendment phase, every reviewed method uses the standard Mahalanobis model (or close one). In this case, gray-level information is collected for each landmark $l$ in all training set images as voxel segments $\left\{g_{i, j}\right\}$ (with $i \in\left[1, n_{s}\right], j \in$ $\left[1, n_{l}\right]$ ) of length $l_{g}=1+2 l_{\text {half }}$, following normal direction to the delineated surface. Mean segments $\overline{g_{l}}$ and covariance matrix $S_{l}$ can then be computed.

When delineating a patient $M r i_{\lambda}$, voxel segments $G_{l}$ of length $l_{G}>>l_{g}$ are also collected around posed shape landmarks. Moves proposals are formulated in selecting the position of $\overline{g_{l}}$ onto $G_{l}$ minimizing the Mahalanobis distance $\left(G_{l}-\overline{g_{l}}\right)^{T} S_{l}^{-1}\left(\overline{g_{l}}-G_{l}\right)$, namely showing highest similarity with the training set learned gray-level environment.

\subsection{A New Intensity Model}

Since our estimated training set forbids accurate determination of $\overline{g_{l}}$ and $S_{l}$, we had to design a new model free from training set priors in focusing on "subtle" intensity variations occuring at structure contours. We also expect better results since, besides intensity normalization problems, the standard approach mainly seeks for best similarity with a learned intensity context.

On segment $G_{l}$, we consider several boundary hypothesis $h$ centered around landmark $l$ position, and for each we retrieve left and right subsegments of constant length $l_{\text {half }}$ : leftmost voxels are supposed to lie inside the structure and rightmost outside. From now on, we assume that finding the boundary consists in choosing $h$ for which voxels of both subsegments will appear most dissimilar.

From other approaches, we noticed that image variability and noise did not let a single intensity-derived measure find the boundary in most cases. Thus we will attempt to find multiple criterions that separately proved to work in - at least - some favorable cases, expecting their combination to raise quite reliable results in most cases. Since we are looking for discontinuities, we will focus on peak values when examining measures.

For image validation purposes, focus was set on a given $M r i_{\lambda}$ and a mean posed model of left Putamen, an "averagely difficult" structure for noise and contrast at borders. Figure 2 display success rates of measures for our case study; after a long sequence of tests, here are those which eventually proved relevant:

- Intensity difference: (Fig 1, green) Though expecting boundaries at maximal intensity differences between the 2 in/out voxels seems a fair assumption, it is not very accurate (33\% success) even if it generally helps anyway.

- Means difference: (dark blue) The absolute difference between inner and outer intensity means should be maximal at boundary, assuming anatomical tissues should be quite different (50\%).

- Inner voxels regularity: (pink) We can also expect homogeneity of anatomical structures: minimization of inner standard deviation finally proves relevant (64\%).

\section{- Outer/Inner regularity difference:}

(light blue) As a corollary, high degree of outer inhomogeneity is expected, hence maximization of outer and inner standard deviation differences $(70 \%)$.

Combining these different measures first requires a common normalization, trivially performed by framing each measure values between 0 and 1. A boundary likelihood (Fig 1, bold red) is then estimated in adding these separate measures, then normalizing the sum, and finally selecting highest likelihood peak. In Fig1, it is reached at hypothesis -1, suggesting a landmark move of 1 voxel inside (visual checking on $M r i_{\lambda}$ confirms this boundary hypothesis).

Coefficients can also be applied to each measure so as to emphasize the most trustful ones. Furthermore, since normalization is very sensitive to min and max values, it is very helpful to discard unlikely hypothesis. These concern land- 
mark positions for which boundary voxel intensity has an outlier value regarding the current inner segment voxels. Also, when outliers are in majority beyond an hypothesis $h$, or when a "wall" of 3 consecutive outliers is found - too much for an occasional artifact, we consider we certainly left the inner structure area and discard upcoming hypothesis.

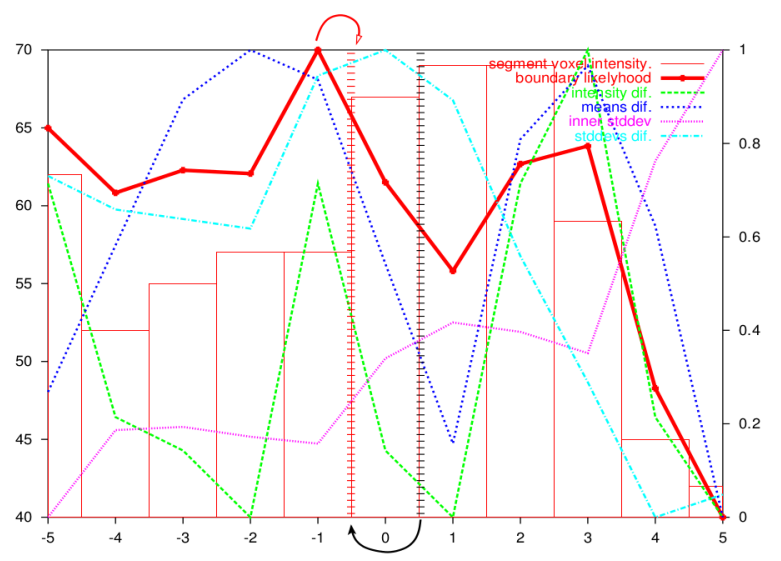

Fig. 1. boundary likelihoods along normal of landmark 1. Highest peak suggests a left move from current model position (black).

Though simplistic at first view, this method proved to be reliable (cf results), since it can adapt to low intensity differences thanks to a strong focus on local discontinuities.

\section{PRELIMINARY RESULTS}

PDMs have been built from 30 MRIs for left/right instances of Caudate Nuclei, Putamens, Thalami and Hippocampi, the ventricles being delineated by our preliminary method.

Our case study Putamen PDM seems correct, since it shows fast decreasing in eigenvalues ( $80 \%$ on first 8 modes) and since associated principal eigenvectors only generate valid shapes (cf fig. 3). Also, the standard deviation around each landmark is about only $3 \%$ of shape bounding box diagonal. The new intensity model proved to be very accurate for our case study, with a critical miss rate (3+ voxels) of $10 \%$, a moderate miss (2) of $15 \%$, and raising the "eye" delineation (miss of $0 / 1$ ) in $75 \%$ of cases. Preliminary tests suggest that similar results can be expected on other structures and MRIs, which is encouraging considering the ASM procedure mostly needs move directions and compensates errors with the PDM.

\section{CONCLUSION AND PERSPECTIVES}

We engaged deliberate efforts to design an automatic delineation method depending only on a single brain atlas an a set of patient MRIs, namely data reasonably available.

\begin{tabular}{|c|c|c|c|c|}
\hline$\%$ & $\begin{array}{l}\text { intensity } \\
\Delta\end{array}$ & $\begin{array}{l}\text { means } \\
\Delta\end{array}$ & $\begin{array}{l}\text { inner } \\
\text { std-dev }\end{array}$ & $\begin{array}{l}\text { outer/inner } \\
\text { std-dev } \Delta\end{array}$ \\
\hline 1st peak & 33 & 50 & 64 & 45 \\
\hline lower peak & 45 & 33 & 11 & 33 \\
\hline critical miss & 22 & 17 & 25 & 22 \\
\hline
\end{tabular}

Fig. 2. success rates for each normalized measure
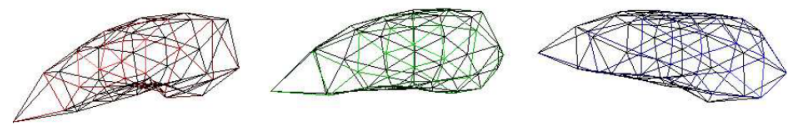

Fig. 3. First variation mode of Left Putamen.

The required statistical shape model is built automatically and will be soon used in conjunction with our suitable intensity model to achieve segmentation results[3]. With the help of praticians, a larger validation campaign is eventually planned.

\section{ACKNOWLEDGMENTS}

We thank Cyceron center for providing numerous brain MRI samples, and also Allan Reinhold Kildeby and Rasmus Larsen (IMM, Copenhagen) who granted us access to their automatic annotation program[8] and their helpful advice.

\section{REFERENCES}

[1] J. Bailleul, S. Ruan, and D. Bloyet. Automatic statistical shape model building from a priori information. In ICISP 2003, volume 2, pages 540-547, Agadir, Morocco, June 2003. IAPR.

[2] I. Bloch, T. Géraud, and H. Maître. Representation and Fusion of Heterogeneous Fuzzy Information in the 3D Space for Model-Based Structural Recognition - Application to 3D Brain Imaging. Artificial Intelligence Journal, 148:141-175, 2003.

[3] D. Cooper, T. Cootes, C. Taylor, and J. Graham. Active shape models - their training and application. Computer Vision and Image Understanding, (61):38-59, 1995.

[4] R. H. Davies. Learning shape: optimal models for analysing natural variability. $\mathrm{PhD}$ thesis, University of Manchester, Division of Imaging Science and Biomedical Engineering, 2002. Supervisor: Chris Taylor.

[5] A. Frangi, D. Rueckert, J. Schnabel, and W. Niessen. Automatic 3d asm construction via atlas-based landmarking and volumetric elastic registration. In M. Insana and R. Leahy, editors, Information Processing in Medical Imaging, volume 2082, pages 78-91, 2001.

[6] A. Hill, A. Thornham, and C. Taylor. Model-based interpretation of $3 \mathrm{~d}$ medical images. In Springer-Verlag, editor, British Machine Vision Conference, pages 339-348, 1992.

[7] A. Kelemen, G. Szekely, and G. Gerig. Elastic model-based segmentation of 3-d neuroradiological data sets. IEEE Transactions on Medical Imaging, 18(10):828-839, october 1999.

[8] A. R. Kildeby. Building optimal 3d shape models. Master's thesis, Informatics and Mathematical Modelling, Technical University of Denmark, DTU, 2002. Supervisor: Rasmus Larsen.

[9] J.-H. Xue, S. Ruan, B. Moretti, M. Revenu, and D. Bloyet. Knowledge-based segmentation an labeling of brain structures from mri images. Pattern Recognition Letters, 22:395-405, 2001. 